\title{
Structure-function analysis of Eiger, the Drosophila TNF homolog
}

Cell Research (2009) 19:392-394. doi: 10.1038/cr.2009.16; publised online 17 February 2009

\section{Dear Editor,}

Tumor necrosis factor (TNF) family ligands in vertebrates are type II transmembrane proteins with functions in the regulation of immunity, bone homeostasis and more [1]. Drosophila expresses a single TNF homologue, Eiger [2, 3], which contains in addition to the homotrimeric C-terminal TNF homology domain (THD) an extended extracellular portion harboring potential protease cleavage sites (Figure 1A-i). One of these sites (Val 145) was identified experimentally [4]. A second potential cleavage site (Arg 211) resembling a canonical furin consensus sequence (R-K-S-R compared to R-X-R/K-R) was cleaved by a furin-like activity when expressed in mammalian 293T cells (Supplementary information, Figure S1). Eiger modulates host responses to Salmonella infections and resistance against extracellular pathogens in Drosophila [5, 6]. When expressed in the eye, it induces cell death via a well-defined pathway (Figure 1B) $[2,3,7]$. The structural requirements for Eiger's activity are however unknown.

As reported previously [3], full-length Eiger expressed in the Drosophila eye under the control of GMR-Gal4 induced a severe apoptotic eye phenotype (Figure 1A-i and $1 \mathrm{C}-\mathrm{i}$ ). Expression of the entire extracellular domain (ecto-Eiger-60) also resulted in complete eye loss (Figure 1A-ii and $1 \mathrm{C}$-iv). As anticipated, the THD that contains the receptor-binding domain in all vertebrate TNF ligands [8] was required for the activity (Figure 1A-vii and 1A-viii). However, shorter forms of soluble Eiger containing the THD alone (Figure $1 \mathrm{~A}-\mathrm{v}$ and $1 \mathrm{~A}-\mathrm{vi}$ ) or corresponding to predicted processed forms (Figure 1A-iii and $1 \mathrm{~A}$-iv) were inactive despite protein expression, and also failed to interfere with death induced by full-length Eiger or ecto-Eiger-60 (data not shown). Moreover, Eiger

Correspondence: Konrad Basler

Tel: +41-44-635-3111; Fax: +41-44-635-6864

E-mail: basler@molbio.uzh.ch lacking the stalk also failed to induce apoptosis (Figure 1A-ix). All these data establish both the THD and the membrane-proximal portion of the stalk (sequence 60145 ) as essential determinants of Eiger's activity.

The JNK pathway mediates the apoptotic activity of Eiger [2, 3, 7] (Figure 1B). Accordingly, the apoptotic activity of ecto-Eiger-60 was totally inhibited when expressed in the eye of $d T A K 1$ hemizygous mutant flies (Figure 1C-v), similar to full-length Eiger expression (Figure 1C-ii). Interestingly, a novel "hanging eye" phenotype was observed when ecto-Eiger-60 was coexpressed in the eye with Puckered, a cell-autonomous inhibitor of Basket [9] (Figure 1C-vi and 1C-vi'). This phenotype, which was not observed when full-length Eiger was co-expressed with Puckered [3] (Figure 1C-iii and $1 \mathrm{C}$-iii'), suggests that soluble Eiger (ecto-Eiger-60) can kill the surrounding tissue that is not protected by Puckered. This was confirmed by clonal analysis in the Drosophila wing imaginal disc. In this experiment, GFPpositive clones co-expressing ecto-Eiger-60 and Puckered induced caspase- 3 activation in neighbouring cells where Puckered was not overexpressed (Figure 1D-iv, 1D-v and 1D-vi). In contrast, full-length Eiger failed to induce obvious activation of caspase-3 within or outside the clone when co-expressed with Puckered (Figure 1Di, 1D-ii and 1D-iii). These results demonstrate that both full-length Eiger and ecto-Eiger-60 induce a JNK-mediated cell death, but that soluble Eiger is able to act over longer distances.

We conclude from these experiments that soluble processed forms of Eiger, if produced at all in the fly, are unlikely mediators of cell death and that the membrane-proximal portion of Eiger may contain a novel functional domain not previously described within the TNF family.

\section{Acknowledgments}

We thank A Tardivel (Department of Biochemistry, University of Lausanne, Switzerland) for expert experimental help and $G$ 
A Expressed proteins

i FL-Egr

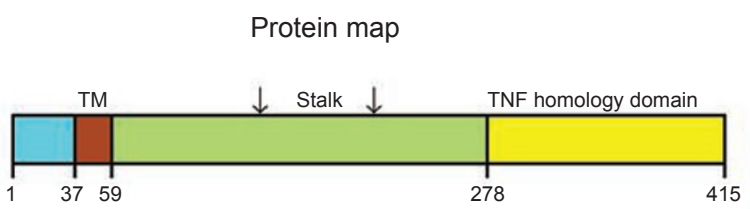

ii Ecto-Egr-60

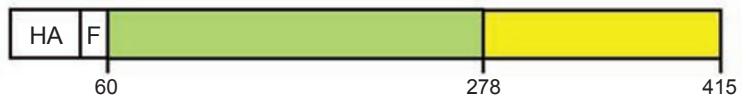

iii Ecto-Egr-146

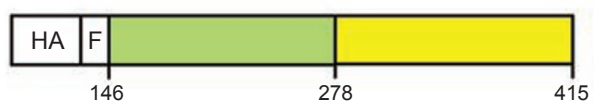

iv Ecto-Egr-212

v Ecto-Egr-265

vi Ecto-Egr-278
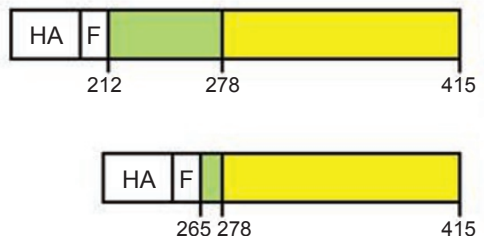

vii

FL-Egr- $\Delta \mathrm{THD}$

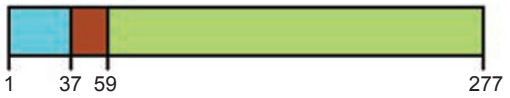

viii Ecto-Egr-60-

$\triangle T H D$

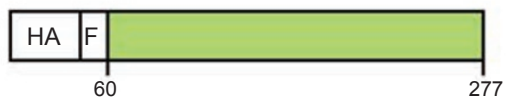

ix FL-Egr$\Delta$ Stalk

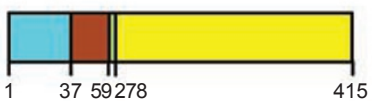

C i
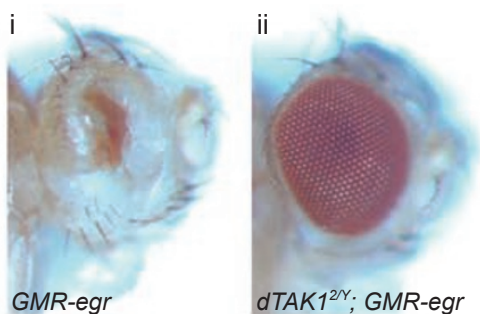

iii
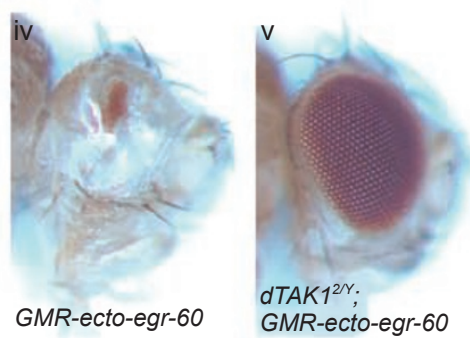
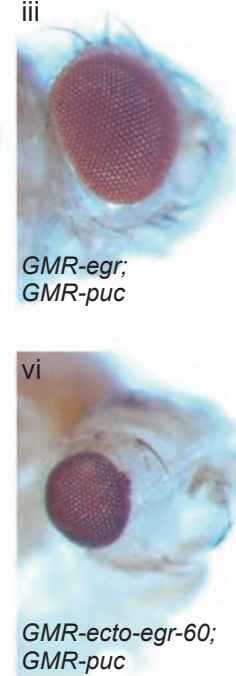

Crossed to GMR-G4
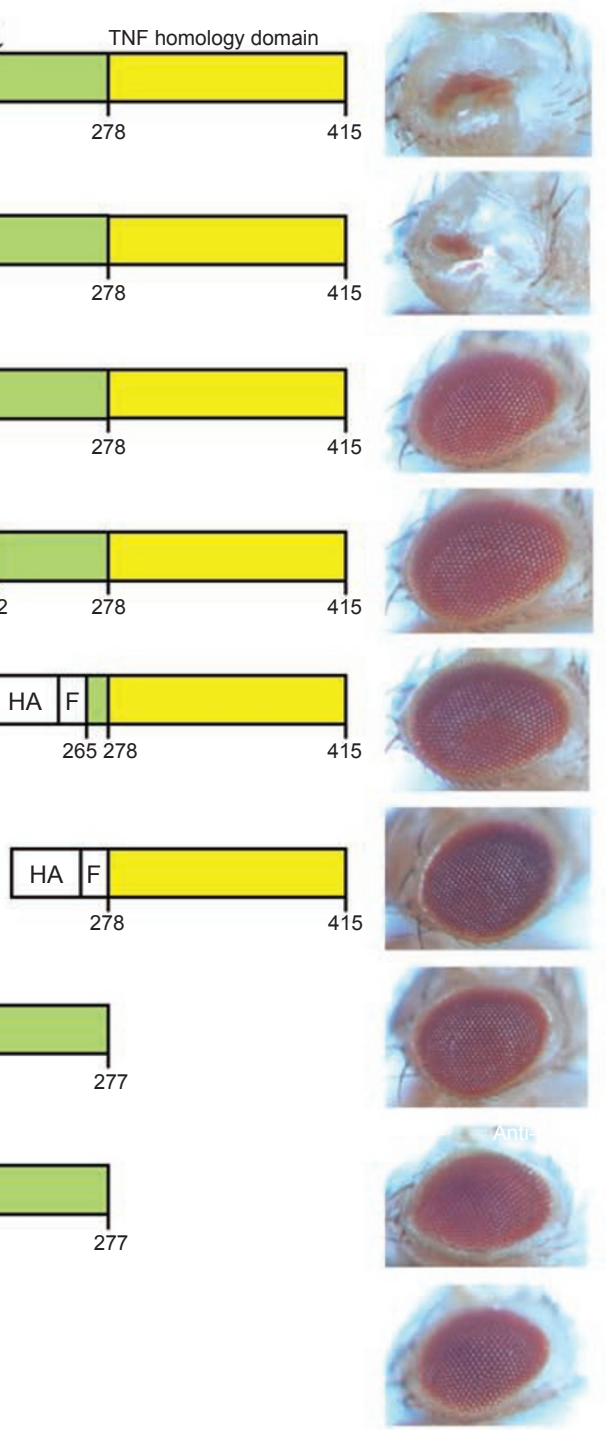

iii' $^{\prime}$

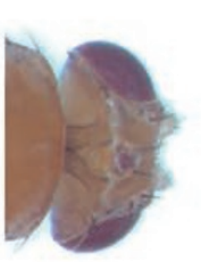

$\mathrm{vi}^{\prime}$

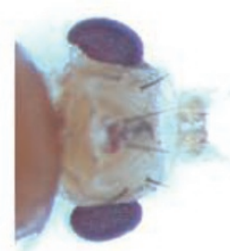

B

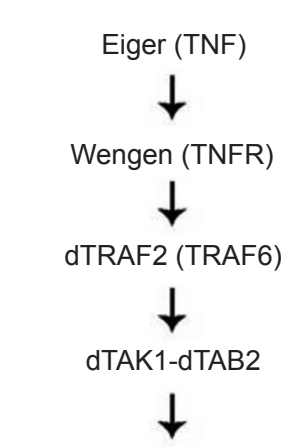

Hemipterous (JNKK)

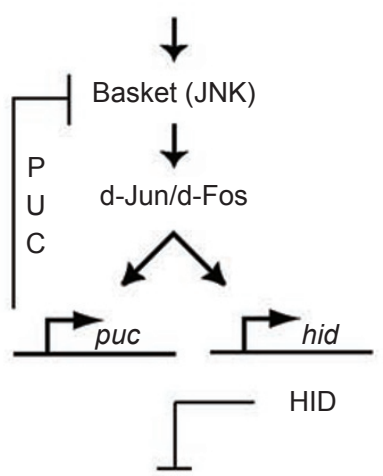

DIAP1 (IAP)

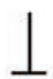

Apoptosome DARK+DRONC (Apaf1+Caspase-9)

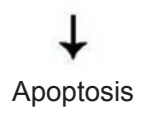


Hausmann (Institute of Molecular Biology, University of Zurich, Switzerland) for insightful comments. This work was supported by the canton of Zurich (to KB), Switzerland and the Swiss National Science Foundation (to KB and PS), Switzerland.

Rajesh Narasimamurthy ${ }^{1}$, Peter Geuking ${ }^{1}$, Karine Ingold $^{2}$, Laure Willen ${ }^{2}$, Pascal Schneider ${ }^{2}$, Konrad Basler $^{1}$

${ }^{1}$ Institute of Molecular Biology, University of Zurich, Winterthurerstrasse 190, 8057 Zurich, Switzerland; ${ }^{2}$ Department of Biochemistry, University of Lausanne, Chemin des Boveresses 155, 1066 Epalinges, Switzerland

\section{References}

1 Locksley RM, Killeen N, Lenardo MJ. The TNF and TNF receptor superfamilies: integrating mammalian biology. Cell 2001; 104:487-501.

2 Igaki T, Kanda H, Yamamoto-Goto Y, et al. Eiger, a TNF superfamily ligand that triggers the Drosophila JNK pathway. EMBO J 2002; 21:3009-3018.
3 Moreno E, Yan M, Basler K. Evolution of TNF signaling mechanisms: JNK-dependent apoptosis triggered by Eiger, the Drosophila homolog of the TNF superfamily. Curr Biol 2002; 12:1263-1268.

4 Kauppila S, Maaty WS, Chen P, et al. Eiger and its receptor, Wengen, comprise a TNF-like system in Drosophila. Oncogene 2003; 22:4860-4867.

5 Brandt SM, Dionne MS, Khush RS, Pham LN, Vigdal TJ, Schneider DS. Secreted bacterial effectors and host-produced Eiger/TNF drive death in a Salmonella-infected fruit fly. PLoS Biol 2004; 2:e418.

6 Schneider DS, Ayres JS, Brandt SM, et al. Drosophila eiger mutants are sensitive to extracellular pathogens. PLoS Pathog 2007; 3:e41.

7 Geuking P, Narasimamurthy R, Basler K. A genetic screen targeting the tumor necrosis factor/Eiger signaling pathway: identification of Drosophila TAB2 as a functionally conserved component. Genetics 2005; 171:1683-1694.

8 Bodmer JL, Schneider P, Tschopp J. The molecular architecture of the TNF superfamily. Trends Biochem Sci 2002; 27:19-26.

9 Martin-Blanco E, Gampel A, Ring J, et al. Puckered encodes a phosphatase that mediates a feedback loop regulating JNK activity during dorsal closure in Drosophila. Genes Dev 1998; 12:557-570.

(Supplementary information is linked to the online version of the paper on the Cell Research website.)

Figure 1 Mapping of functional domains of Eiger. (A) Schematic representation of expression constructs used in this study. HA, hemagglutinin signal peptide; F, Flag tag and linker; $\downarrow$, predicted cleavage sites at amino acid position 145 and 211. Numbers refer to the amino acid sequence of Eiger. The eye phenotypes generated by transgenic expression of the corresponding Eiger constructs are shown. (B) Schematic representation of the Eiger signalling pathway. Protein names in brackets refer to the mammalian homologue. (C) Long-range diffusion and death-inducing activity of soluble ecto-Eiger-60. The indicated forms of Eiger were expressed in the eye of wild-type control (i and iv) or $d T A K 1$-deficient flies (ii and v) (dTAK $1^{2} / Y$, where $d T A K 1^{2}$ is an inactive mutant allele of dTAK1), or in flies overexpressing Puckered in the eye (UAS-puc) (iii and vi; iii' and vi' show the eye phenotypes of iii and vi from the top). (D) The indicated form of Eiger was co-expressed with Puckered and GFP (by crossing y w hs-flp; actin>CD2>Gal4 UAS-GFP flies with flies carrying UAS-egr; UAS-puc or UAS-ecto-egr-60; UAS-puc). Clones of GFP-positive cells (in green) were analyzed in the wing imaginal disc. Cell death was detected by anticaspase-3 staining (in red). (See supplementary information, Data S1, for the experiments described.) 